\title{
Incorporação de nitrogênio e fósforo no tecido foliar da macrófita Typha domingensis Pers. durante o tratamento de efluente da bovinocultura leiteira em wetlands construídos
}

\author{
Incorporation of nitrogen and phosphorus in the \\ leaves of Typha domingensis Pers. macrophyte during \\ treat dairy cattle wastewater in constructed wetlands
}

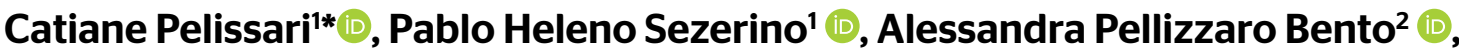
Orlando de Carvalho Junior ${ }^{3}\left(0\right.$, Samara Terezinha Decezaro ${ }^{4} \oplus$, Delmira Beatriz Wolff ${ }^{4}$

口

\section{RESUMO}

O objetivo deste estudo foi avaliar o desempenho da macrófita Typha domingensis Pers., em diferentes etapas de seu desenvolvimento, na remoção de nitrogênio $(N)$ e fósforo $(P)$ em um wetland construído horizontal (WCH) aplicado no tratamento de efluente de bovinocultura leiteira. Para isso, foi realizado o monitoramento do crescimento e dos teores de $\mathrm{N}$ e $\mathrm{P}$ no tecido foliar das macrófitas, durante um período de 120 dias de crescimento. A macrófita Typha domingensis Pers. foi responsável por uma remoção média de 5,12 e 3,16\% das cargas de N e $\mathrm{P}$ aplicadas no WCH, respectivamente. As maiores taxas de remoções de nutrientes (30,2 e 6,4 g P kg-1) foram identificadas quando ocorreram as maiores taxas de crescimento foliar (24,17 cm semana').

Palavras-chave: wetland construído; nitrogênio; fósforo; taxa de crescimento.

\begin{abstract}
The objective of this study was to evaluate the performance of macrophyte Typha domingensis Pers. in different development stages in the nitrogen and phosphorus removal from a horizontal flow constructed wetland (WCH) applied for dairy cattle wastewater treatment. In this way, growth, nitrogen and phosphorus levels in the leaf tissue of macrophytes were monitored during a 120-day growth period. Typha domingensis Pers. macrophyte was responsible for a mean removal of 5.12 and $3.16 \%$ of the applied loads in the WCH, for nitrogen and phosphorus, respectively. The higher nutriens removal rates (30.2 and $6.4 \mathrm{~g} \mathrm{P} \mathrm{kg}^{-1}$ ) were identified when the higher tissue growth rates occurred (24.17 $\mathrm{cm}$ week')
\end{abstract}

Keywords: constructed wetland; nitrogen; phosphorus; growth rate.

\section{INTRODUÇÃO}

Os wetlands construídos (WC) são sistemas amplamente utilizados para o tratamento de diversos tipos de efluentes, tais como domésticos, industriais e agrícolas. São conhecidos como tecnologia de baixo custo de implantação, operação e manutenção, e estão em constante desenvolvimento em todo o mundo. Além disso, essa tecnologia vem demonstrando boa adaptação nos mais variados cenários, apresentando-se como um sistema naturalmente integrado ao ambiente, sendo atrativo na manutenção dos aspectos paisagísticos.
Entre as modalidades de WC, o wetland construído horizontal (WCH) pode atingir eficiência de tratamento em nível secundário e avançado. Essa modalidade de WC pode ser uma boa alternativa quando busca-se tratar o efluente em locais povoados ou em áreas recreativas, em razão, principalmente, da ausência de aspersão de esgoto por bombeamento (evitando formação de aerossóis), bem como da inexistência de insetos (GARCÍA et al., 2010). Nesses sistemas, a depuração das águas residuárias ocorre por meio da interação do material filtrante, os micro-organismos e as macrófitas (KADLEC \& WALLACE, 2009). 
Atualmente, o papel das macrófitas como um componente essencial nos WC está bem estabelecido, uma vez que elas atuam diretamente nos mecanismos de remoção de poluentes (BRIX, 1997; KADLEC \& WALLACE, 2009). A assimilação de nutrientes, apesar de ser em baixa magnitude, é uma das principais atribuições dadas às macrófitas nessa tecnologia de tratamento. No Brasil, vários estudos já foram conduzidos a fim de quantificar a assimilação de nutrientes pelas macrófitas em WC (BRASIL et al., 2007; MATOS et al., 2008; 2010; FIA et al., 2008; 2011).

No entanto, a assimilação de nutrientes pelas macrófitas está associada a vários fatores, como a modalidade de WC, as cargas orgânicas e inorgânicas aplicadas no sistema, as condições ambientais, bem como a espécie empregada (SAEED \& SUN, 2012). Recentemente, um estudo mostrou que, no Brasil, Typha spp (popularmente conhecida como taboa) se destaca como a espécie de macrófita mais empregada em WCH (SEZERINO et al., 2015).

Além disso, estudos mostraram que, para efluente proveniente de bovinocultura leiteira, os WCH apresentaram desempenho de tratamento satisfatório (PELISSARI et al., 2014). No entanto, pouco se conhece sobre a magnitude de assimilação de nutrientes pela macrófita Typha associado a esse tipo de efluente. Ademais, o mecanismo de assimilação de nutrientes ao longo das diferentes fases do crescimento da macrófita Typha ainda não está elucidado. Nesse contexto, o objetivo deste estudo foi avaliar o desempenho de remoção de nitrogênio (N) e fósforo (P) pela macrófita Typha domingensis Pers., em um WCH empregado para o tratamento de efluente de bovinocultura leiteira.

\section{METODOLOGIA}

\section{Descrição da estação de tratamento de efluente}

O estudo foi conduzido nas dependências de uma instalação de bovinocultura leiteira com capacidade de produção diária de $140 \mathrm{~L}$ de leite, situada na cidade de Frederico Westphalen, Rio Grande do Sul (altitude $27^{\circ} 21^{\prime} 33^{\prime \prime}$ sul, longitude $53^{\circ} 23^{\prime} 40^{\prime \prime}$ oeste e $566 \mathrm{~m}$ de altitude). A planta de tratamento de efluente produzido nas instalações da bovinocultura leiteira era composta por uma lagoa de armazenamento (LA), a qual atuou como tratamento primário e recebeu todo o volume de efluente produzido, e um WCH operado em série. Parte do efluente da LA era conduzida por gravidade para o WCH, que, após o tratamento, era infiltrado no solo por meio de valas de infiltração (Figura 1).

$\mathrm{O} \mathrm{WCH}$ tinha uma área superficial de $26,5 \mathrm{~m}^{2}$, plantado com a macrófita Typha domingensis Pers (plantadas em uma razão de 4,5 plantas por $\mathrm{m}^{2}$ ), e areia grossa (diâmetro efetivo $\mathrm{d}_{10}=0,3 \mathrm{~mm}$ e uniformidade $=2,5)$ como material filtrante. A vazão média diária de efluente aplicada no WCH foi de $995 \mathrm{~L}^{-1}$, distribuída ao longo de 4 horas do dia (das 9 às 13h), resultando em uma taxa hidráulica aplicada de $37,5 \mathrm{~mm}$ $\mathrm{d}^{-1}$. O regime hidráulico operado no WCH foi intercalando ciclos de alimentação e repouso de 4 e 3 dias, respectivamente, ao longo da semana.
Durante o período do estudo (maio a outubro de 2012), análises das concentrações de $\mathrm{N}$ total e $\mathrm{P}$ ortofosfato do afluente e do efluente do WCH foram realizadas uma vez por semana, segundo recomendações de American Public Health Association (APHA, 2005).

\section{Monitoramento do crescimento e assimilação de nitrogênio e fósforo das macrófitas}

Para monitorar o crescimento das macrófitas, foi realizada a poda de todos os indivíduos presentes no $\mathrm{WCH}$, em uma altura de $30 \mathrm{~cm}$ acima da superfície do material filtrante. Em seguida, o WCH foi dividido em seis quadrantes (Figura 2) para a obtenção de amostras aleatórias

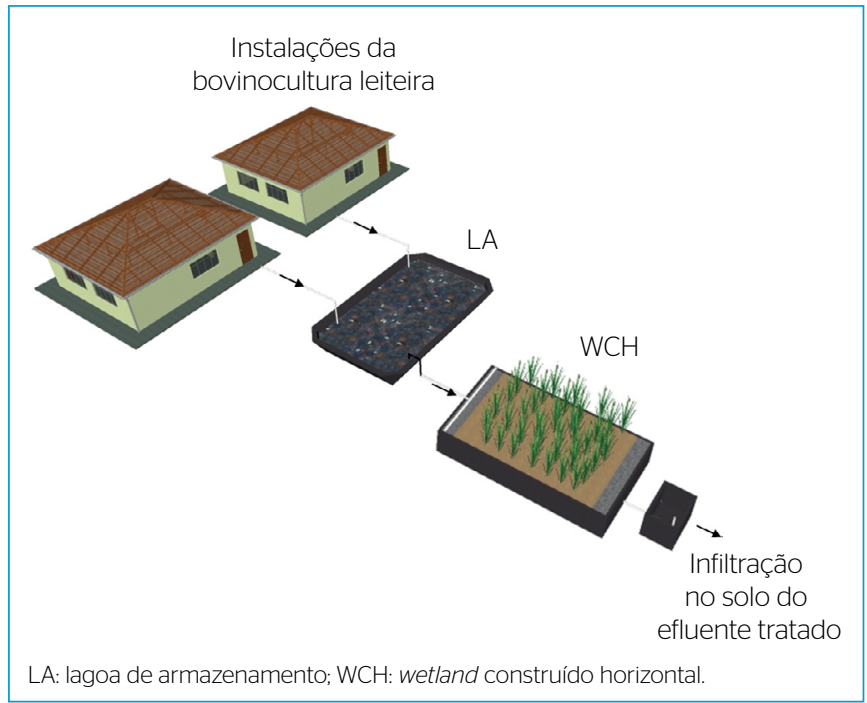

Figura 1 - Diagrama da planta de tratamento de efluente implantada nas instalações da bovinocultura leiteira.

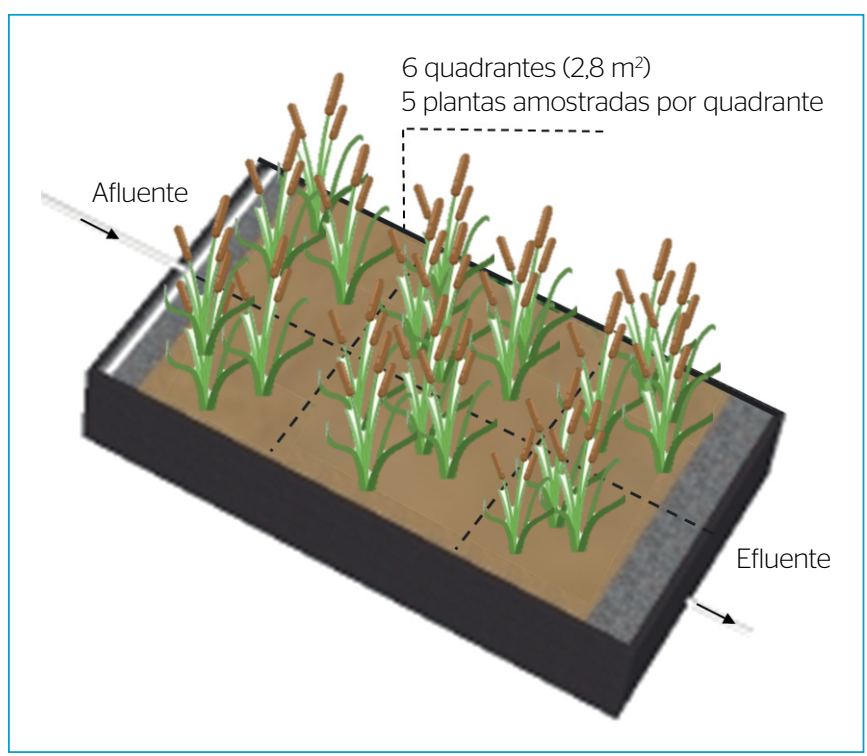

Figura 2 - Esquema representando a área superficial do wetland construído horizontal dividido em quadrantes. 
e estatisticamente representativas da biomassa vegetal emergente no sistema. O acompanhamento do crescimento das macrófitas foi feito semanalmente, a partir da poda. Para tanto, realizou-se um sorteio aleatório de cinco plantas por quadrante, as quais foram identificadas ao longo do período amostrado. As medições de crescimento deram-se com o auxílio de fita métrica. A taxa de crescimento das macrófitas amostradas foi calculada, seguindo recomendações de Esteves (1998), conforme descrito na Equação 1.

$T A=\left(\frac{C^{2}}{T^{2}}-\frac{C^{1}}{T^{1}}\right)$

Em que:

$\mathrm{TA}=$ taxa de crescimento $\left(\mathrm{cm} \mathrm{d}^{-1}\right)$;

$\mathrm{C}_{2}$ e $\mathrm{C}_{1}=$ comprimento médio das folhas nos tempos 1 e $2(\mathrm{~cm})$;

$\mathrm{T}_{2}-\mathrm{T}_{1}=$ intervalo entre as coletas (dias).

Para avaliar os teores de N e P no tecido vegetal das macrófitas, foi coletada 1 planta por quadrante do $\mathrm{WCH}$, em diferentes fases do crescimento das macrófitas $(44,58,92,105,133$ e 150 dias de crescimento), totalizando 6 plantas amostradas por fase de crescimento. Após a coleta, a parte áerea das macrófitas foi levada à estufa, sob temperatura de $60^{\circ} \mathrm{C}$, até atingir peso constante. Após essa etapa, parte das amostras foi separada para a análise nutricional e o restante das amostras permaneceu na estufa sob temperatura de $105^{\circ} \mathrm{C}$, até atingir peso constante, para a quantificação da massa seca total. As análises dos teores de $\mathrm{N}$ e P no tecido foliar seguiram as recomendações de Tedesco et al. (1995).

Para os cálculos de assimilação de nutrientes pelas macrófitas, foi considerada uma relação entre teor de $\mathrm{N}$ e $\mathrm{P}\left(\mathrm{g} \mathrm{kg}^{-1}\right)$ no tecido foliar, massa seca foliar $(\mathrm{kg})$, crescimento das macrófitas $(\mathrm{cm})$ e sua densidade no filtro (plantas $\mathrm{m}^{-2}$ ). $\mathrm{O}$ número de plantas foi determinado contando todas as plantas, sem distinguir os colmos das emissões laterais (brotos), sendo adotado o conceito de que cada broto corresponde a um indivíduo. Ressalta-se que as cargas de $\mathrm{P}$ assimiladas pelas macrófitas foram baseadas nas cargas de $\mathrm{P}$ ortofosfato aplicadas.

\section{RESULTADOS E DISCUSSÃO}

\section{Desenvolvimento da macrófita Typha domingensis Pers. no wetland construído horizontal}

Decorridos 100 dias de crescimento, as macrófitas atingiram a altura máxima $(207 \mathrm{~cm})$. Após esse período, ocorreu a estabilização do crescimento, concomitantemente ao início da inflorescência e senescência das macrófitas (Figura 3A). Com base nesse comportamento, as maiores taxas de crescimento deram-se no início do ciclo de desenvolvimento das macrófitas, ocorrendo variação entre 1,35 e $24,17 \mathrm{~cm}$ semana $^{-1}$ (Figura 3B). Esse mesmo comportamento já foi relatado por outros estudos realizados em WCH, em que ocorreu maior crescimento da Typha domingensis Pers. na primeira semana após o plantio (crescimento médio de $19 \mathrm{~cm}$ ); após esse período, a altura média identificada foi de $130 \mathrm{~cm}$ com 90 dias de crescimento e de $210 \mathrm{~cm}$ em um período de 150 dias (SOUZA, 2003).

Além disso, identificou-se maior desenvolvimento das macrófitas na zona de entrada do WCH (quadrantes 1 e 2). Esse fato pode estar associado com a maior disponibilidade de nutrientes sessa seção, pela proximidade desta com a zona de entrada de efluente (Figura 4).

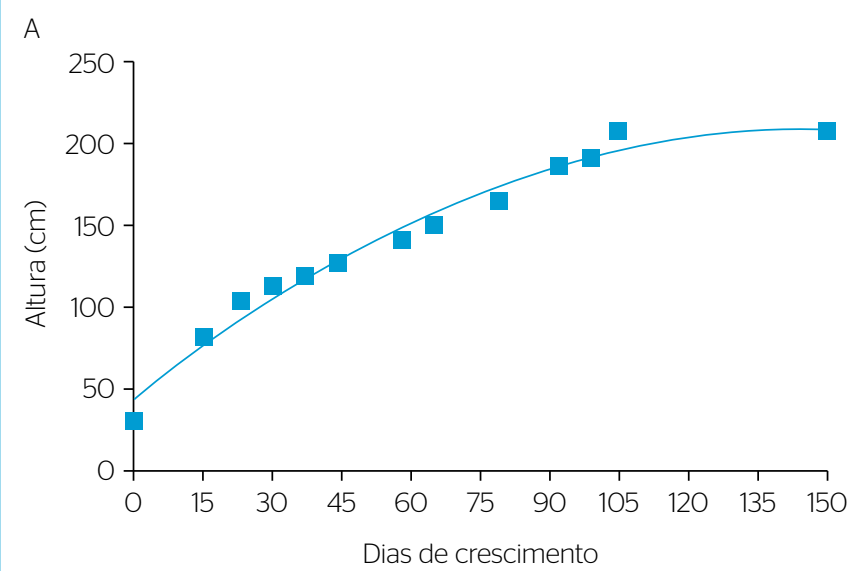




\section{Teores de nitrogênio e fósforo no tecido foliar da macrófita Typha domingensis Pers.}

As maiores concentrações de nutrientes tanto de $\mathrm{N}$ quanto de $\mathrm{P}(30,2 \mathrm{e}$ $\left.6,4 \mathrm{~g} \mathrm{P} \mathrm{kg}^{-1}\right)$ no tecido foliar das macrófitas foram identificadas no início do desenvolvimento das macrófitas, ou seja, próximo de 60 dias de crescimento (Figura 5). Esses valores de concentrações coincidiram com as maiores taxas de crescimento (Figura 3), apresentando diminuição nos teores de nutrientes decorridos cerca de 90 dias de crescimento $(25,5$ e $\left.2,4 \mathrm{~g} \mathrm{P} \mathrm{kg}^{-1}\right)$. Esse fato pode estar associado com a translocação de nutrientes para as gemas reprodutivas das plantas. A maior parte dos nutrientes transcoláveis é produzida durante a passagem da fase vegetativa para a fase reprodutiva de desenvolvimento e durante a mobilização das proteínas estocadas para novos ramos da planta (LARCHER, 2000). Trabalhos conduzidos no Brasil reportam grande variação da concentração de nutrientes no tecido foliar da macrófita Typha domingensis Pers. Brasil et al. (2007), ao utilizarem Typha no tratamento de esgoto doméstico, obtiveram valores de 15 e 2,8 $\mathrm{g} \mathrm{kg}^{-1}$ para N e P, respectivamente, Freitas (2006) verificou valores na ordem de 21,30 $\mathrm{g} \mathrm{K} \mathrm{N} \mathrm{g}^{-1}$ e 4,4 $\mathrm{g} \mathrm{P} \mathrm{Kg}^{-1}$. Em outro trabalho, Fia et al. (2011) encontraram valores variando de 29,90 a 32,7 $\mathrm{g} \mathrm{N} \mathrm{kg}^{-1} \mathrm{e}$ de 2 a 3,2 $\mathrm{g} \mathrm{P} \mathrm{kg}^{-1}$. Essa variação pode estar associada com a disponibilidade de nutrientes no meio, a natureza do efluente e a fase de desenvolvimento das plantas analisadas (SAEED \& SUN, 2012).

\section{Influência da macrófita Typha domingensis Pers. no desempenho de tratamento do wetland construído horizontal em relação à remoção de nitrogênio e fósforo}

As remoções médias de $\mathrm{N}$ e $\mathrm{P}$ foram de 1,07 e 0,17 $\mathrm{g} \mathrm{m}^{-2}$ semana $^{-1}$, respectivamente. Esses valores correspondem a uma remoção média de $5,12 \%$ da carga total de $\mathrm{N}$ e de $3,16 \%$ da carga total de $\mathrm{P}$ aplicadas no WCH (Tabela 1). Esses valores são superiores aos encontrados por Brasil et al. (2007) (1,69\% para N e 1,64\% para P). Como comentado, as

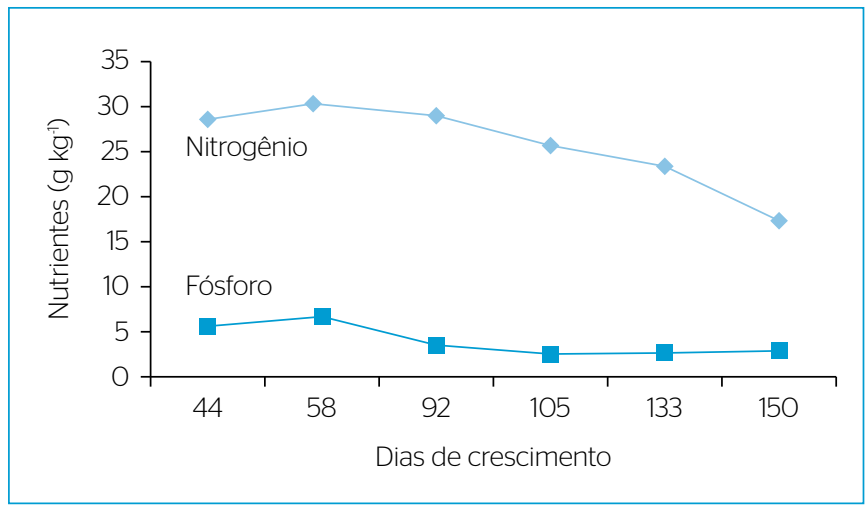

Figura 5 - Concentração de nitrogênio e fósforo no tecido vegetal da macrófita Typha domingensis Pers.
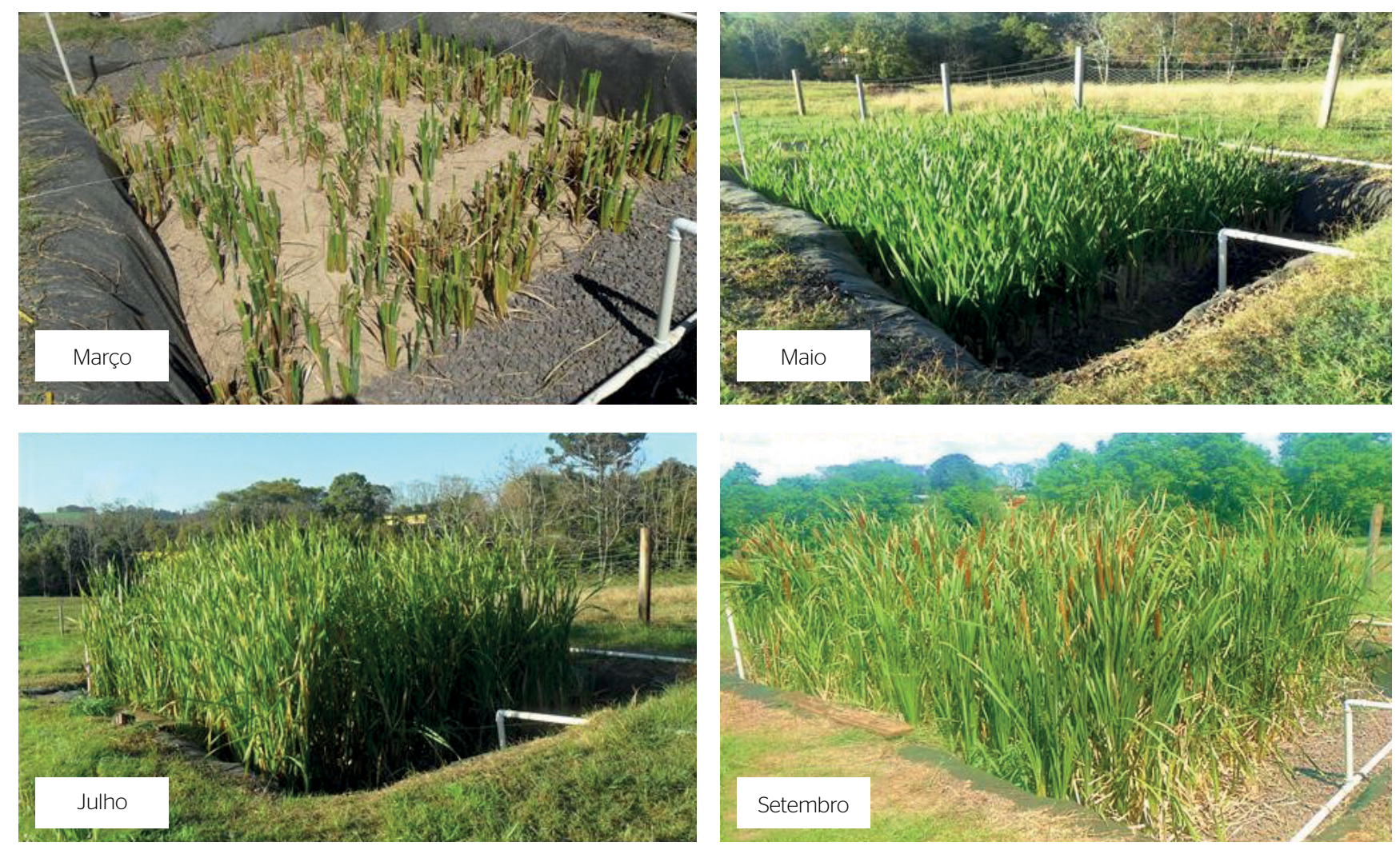

Figura 4 - Desenvolvimento da macrófita Typha domingensis Pers. no wetland construído horizontal ao longo de do período de março a setembro de 2012. 
Tabela 1 - Desenvolvimento e incorporação de nutrientes no tecido foliar da macrófita Typha domingensis Pers. presente no wetland construído horizontal*.

\begin{tabular}{l|c|c|c|c|c|c|c}
$\begin{array}{l}\text { Dias de } \\
\text { crescimento }\end{array}$ & Crescimento (m) & $\begin{array}{c}\text { Carga NT } \\
\text { aplicada (kg) }\end{array}$ & $\begin{array}{c}\text { Carga NT incorporada } \\
\text { tecido vegetal (kg) }\end{array}$ & $\begin{array}{c}\text { N removido } \\
\text { pelo tecido } \\
\text { vegetal (\%) }\end{array}$ & $\begin{array}{c}\text { Carga P-PO }{ }_{4}^{3-} \\
\text { aplicada (kg) }\end{array}$ & $\begin{array}{c}\text { Carga P incorporada } \\
\text { tecido vegetal (kg) }\end{array}$ \\
\hline 0-30 & 0,83 & 2,00 & 0,15 & 7,5 & 0,49 & 0,03 \\
\hline vegetal (\%)
\end{tabular}

*Os cálculos de remoção de nutrientes pelo tecido foliar foram realizados com base na relação massa seca (7,35 g de massa seca por metro de tecido foliar), crescimento e densidade de macrófitas (850 plantas, ou seja, uma densidade de 50 plantas por m² de filtro plantado); NT: nitrogênio total; N: nitrogênio; P: fósforo.

maiores remoções de nutrientes obtidas no presente trabalho, em comparação a outros realizados no Brasil, podem estar relacionadas com as maiores cargas de nutrientes aplicadas, quando comparadas com os demais autores. Além disso, as remoções de nutrientes pelas macrófitas nos WCH dependem, em grande parte, da densidade de plantas e também do clima, o qual influencia na sua taxa de crescimento. É visível que a maior remoção de nutrientes ocorreu no primeiro mês de crescimento das macrófitas, em função das suas maiores taxas de crescimento.

Com base nos resultados obtidos pode-se sugerir que o período ideal para a poda é aquele em que as macrófitas atingem seu máximo desenvolvimento, ou seja, quando se reduzem significativamente as taxas de crescimento, o que no presente estudo se deu entre 60 e 90 dias após a poda, corroborando os estudos de Vera et al. (2010).

\section{CONCLUSÕES}

Por meio do monitoramento do crescimento e dos teores de $\mathrm{N}$ e P no tecido foliar da macrófita Typha domingensis Pers. presentes em um WCH aplicado no tratamento de efluente de bovinocultura leiteira, pode-se concluir:
- A remoção de nutrientes pelas macrófitas no WCH está relacionada com as taxas de crescimento e com a fase de desenvolvimento das plantas;

- Os teores médios de $\mathrm{N}$ e $\mathrm{P}$ no tecido foliar foram de 25,6 e $3,8 \mathrm{~g} \mathrm{~kg}^{-1}$, respectivamente;

- A macrófita foi responsável pela remoção média de 5,12 e 3,16\% das cargas de $\mathrm{N}$ e $\mathrm{P}$ aplicadas no $\mathrm{WCH}$, respectivamente;

- A poda de Typha domingensis Pers. em WCH deve ser realizada quando a macrófita atingir ser crescimento máximo, o que, neste estudo, se deu entre 60 e 90 dias após a poda.

\section{FONTE DE FINANCIAMENTO}

Conselho Nacional de Desenvolvimento Científico e Tecnológico (CNPq).

\section{AGRADECIMENTOS}

Os autores gostariam de agradecer ao Conselho Nacional de Desenvolvimento Científico e Tecnológico (CNPq), o financiamento da pesquisa (Edital MCT/CNPq nº 014/2010).

\section{REFERÊNCIAS}

APHA, AWWA, WEF. Standard methods for the examination of water and wastewater. $21^{\text {st }}$ ed. Washington, DC: American Public Health Association, 2005.

BRASIL, M. da S.; MATOS, A.T. de; SOARES, A.A. (2007) Plantio e desempenho fenológico da taboa (Typha sp.) utilizada no tratamento de esgoto doméstico em sistemas alagados construídos. Engenharia Sanitária Ambiental, v. 12, n. 3, p. 266-272. http://dx.doi.org/10.1590/s141341522007000300006

BRIX, H. (1997) Do macrophytes play a role in constructed treatment wetlands? Water Science and Technology, v. 35, n. 5 , p. 11-17. https://doi.org/10.1016/SO273-1223(97)00047-4
ESTEVES, F.A. Fundamentos de Limnologia. Rio de Janeiro: Interciência/FINEP, 1998. 575p.

FIA, F.R.L.; MATOS, A.T. de; FIA, R.; LAMBERT, T.F.; MATOS, M.P. de (2011) Remoção de nutrientes por Typha latifolia e Cynodon spp. cultivadas em sistemas alagados construídos. Ambiente \& Água, v. 6, n. 1, p. 77-89. https://doi.org/10.4136/ambi-agua.17

FIA, R.; MATOS, A.T. de; FERREIRA, P.A.; TEODORO, P.E.P.; SCHUERY, F.C.; LUIZ, F.A.R. (2008) Desempenho agronômico da Typha sp. e Alternanthera philoxeroides mart utilizadas no tratamento de águas residuárias da lavagem e descascamento/despolpa dos frutos do cafeeiro em sistema alagado construído. Engenharia na Agricultura, Viçosa, v. 16, n. 4, p. 436-448. 
FREITAS, W.S. (2006) Desempenho de sistemas alagados construidos, cultivados com diferentes espécies vegetais, no tratamento de águas residuárias da suinocultura. 159f. Tese (Doutorado) - Universidade Federal de Viçosa, Viçosa.

GARCÍA, J.; ROUSSEAU, D.P.L.; MORATÓ, J.; LESAGE, E.; MATAMOROS, V.; BAYONA, J.M. (2010) Contaminant removal processes in subsurface-flow constructed wetlands: a review. Environmental Science Technology, v. 40, n. 7, p. 561-661. https://doi. org/10.1080/10643380802471076

KADLEC, R.H.; WALLACE, S.D. (2009) Treatment wetlands. 2. ed. Boca Raton: CRC Press. 1016 p.

LARCHER, W. (2000) Ecofisiologia vegetal. São Carlos: Editora RIMA. 519 p.

MATOS, A.T. de; ABRAHÃO, S.S.; MONACO, P.L.V.A.; SARMENTO, A.P.; MATOS, M.P. (2010) Capacidade extratora de plantas em sistemas alagados utilizadas no tratamento de águas residuárias de laticínios. Engenharia Agrícola Ambiental, v. 14, n. 12, p. 1311-1317.

MATOS, A.T. de; ABRAHÃO, S.S.; PEREIRA, O.G. (2008) Desempenho agronômico do capim tifton 85 (cynodon spp) cultivados em sistemas alagados construídos utilizados no tratamento de água residuária de laticínios. Ambiente \& Água, Taubaté, v. 3, n. 1, p. 43-53. https://doi.org/10.4136/ambi-agua.41

PELISSARI, C.; SEZERINO, P.H.; DECEZARO, S.T.; WOLFF, D.B.; BENTO, A.P.; CARVALHO JÚNIOR, O.; PHILIPPI, L.S. (2O14) Nitrogen transformation in horizontal and vertical flow constructed wetlands applied for dairy cattle wastewater treatment in southern Brazil.
Ecological Engineering, v. 73, p. 307-310. http://dx.doi.org/10.1016/j. ecoleng.2014.09.085

SEZERINO, H.P.; BENTO, A.P.; DECEZARO, S.T.; MAGRI, M.E.; PHILIPPI, L.S. (2015) Experiências brasileiras com wetlands construídos aplicados ao tratamento de águas residuárias: parâmetros de projeto para sistemas horizontais. Engenharia Sanitária e Ambiental, v. 20, n. 1, p. 151-158. http://dx.doi.org/10.1590/S141341522015020000096615

SAEED, T.; SUN, G. (2012) A review on nitrogen and organics removal mechanisms in subsurface flow constructed wetlands: dependency on environmental parameters, operating conditions and supporting media. Journal of Environmental Management, v. 112, p. 429-488. https://doi.org/10.1016/j.jenvman.2012.08.011

SOUZA, L.M.I. de. (2003) Avaliação da macrófita Typha domingensis Pers. no pós-tratamento de efluentes do campus da UFMS e do hospital universitário, em banhados construidos de fluxo subsuperficial. Dissertação (Mestrado em Saneamento Ambiental e Recursos Híbridos) - Universidade Federal de Mato Grosso do Sul, Campo Grande.

TEDESCO, M.J.; GIANELLO, C.; BISSANI, C.A.; BOHNEN, H.; VOLKWEISS, S.J. (1995) Análise de solo, plantas e outros materiais. Porto Alegre: Universidade Federal do Rio Grande do Sul. 174 p.

VERA, A.; ANDRADE, C.; FLORES, E.; NÚÑEZ, M.; CÁRDENAS, C.; MORALES, E. (2010) Removal of nutrients and organic matter in a constructed wetland, in function of the development of the macrophyte Typha dominguensis Pers. Revista Técnica de la Facultad de Ingeniería Universidad del Zulia, v. 33, n. 2, p. 153-163. 